Supporting Information

\title{
Silicene Quantum Dots Confined in Few-Layer Siloxene Nanosheets for Blue Light-Emitting Diodes
}

Xinling Xu, Liping Zhou, Degong Ding, Yang Wang, Jingyun Huang, * Haiping He, and

Zhizhen Ye*

School of Materials Science and Engineering, State Key Laboratory of Silicon Materials,

Zhejiang University, Hangzhou 310027, China

*Email: huangjy@zju.edu.cn (JY Huang),yezz@zju.edu.cn(ZZ Ye) 

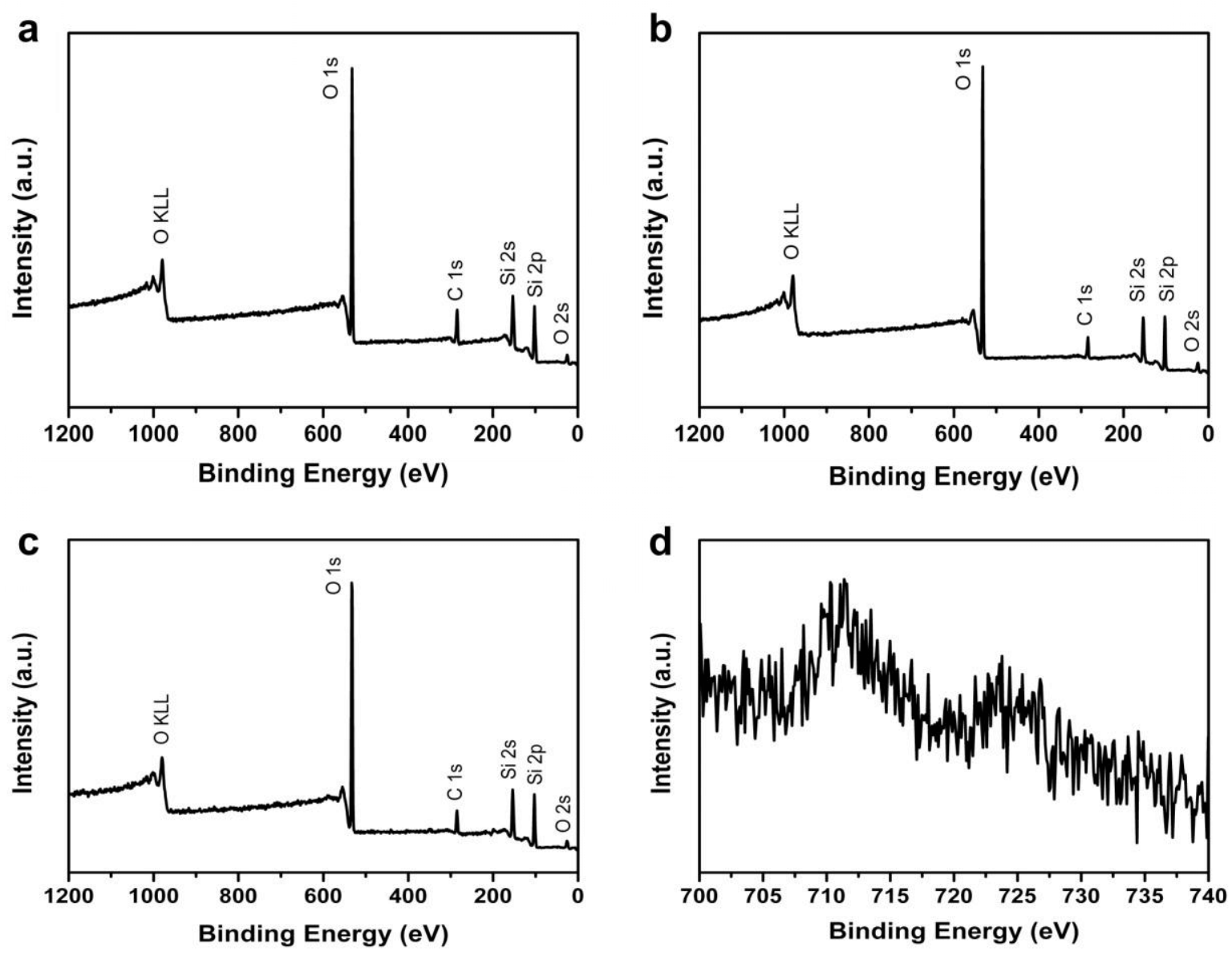

Figure S1. a-c) X-ray photoelectron spectra for full range scan of a) HCl-siloxene, b) EACsiloxene, and c) QD-siloxene, respectively. d) X-ray photoelectron spectra for Fe 2p states of QD-siloxene. 

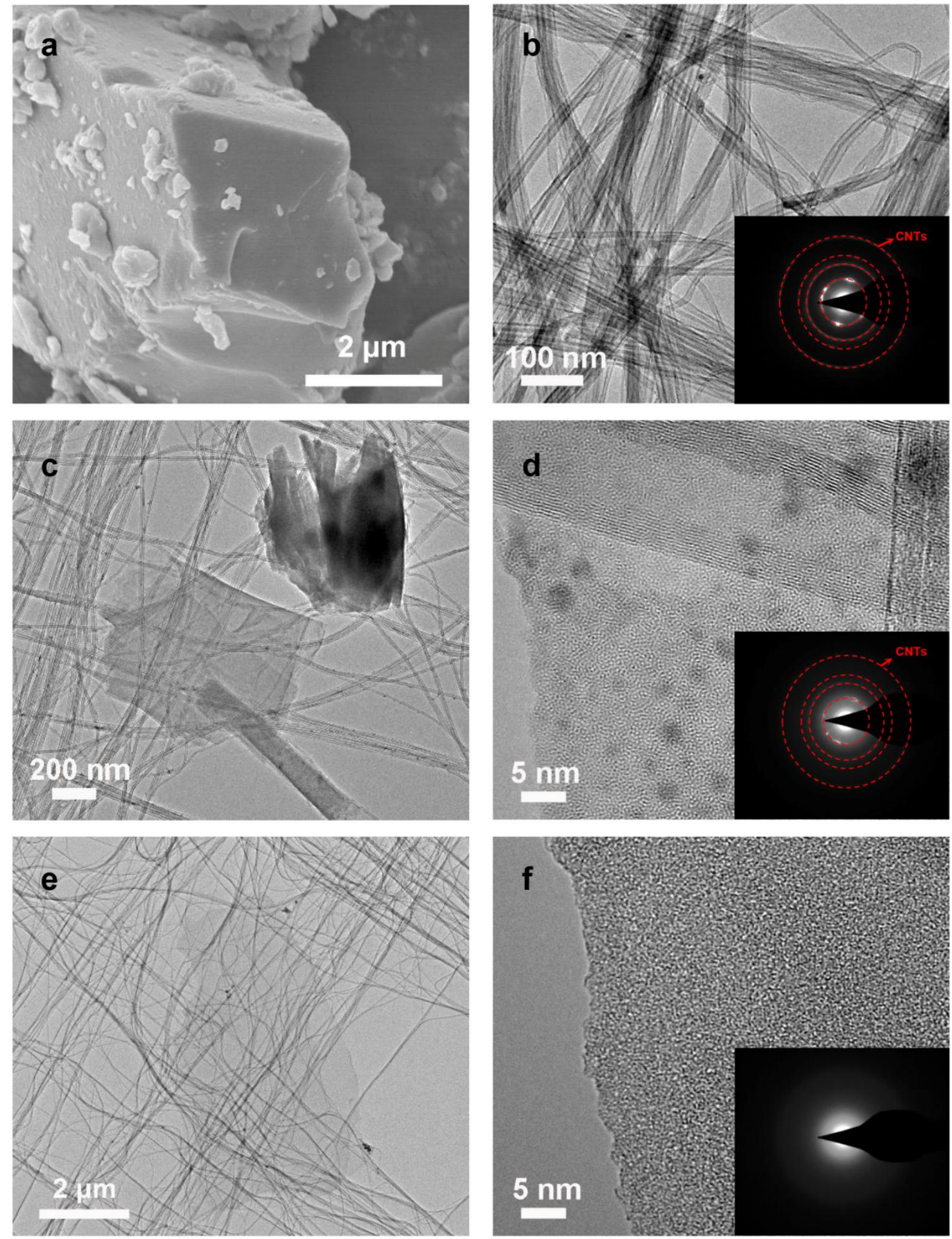

Figure S2. a) SEM image of bulk $\mathrm{CaSi}_{2}$. b) TEM image and SAED pattern of the carbon nanotube (CNT) coated on the TEM grid. c) TEM, d) HRTEM images and SAED pattern of EAC-siloxene. e) TEM, f) HRTEM images and SAED pattern of HCl-siloxene. 


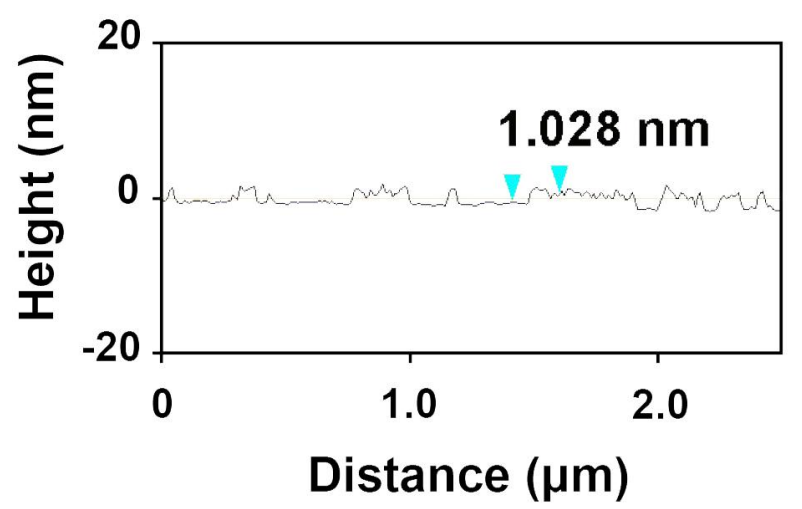

Figure S3. The height profile of QD-siloxene nanosheets in the AFM image.
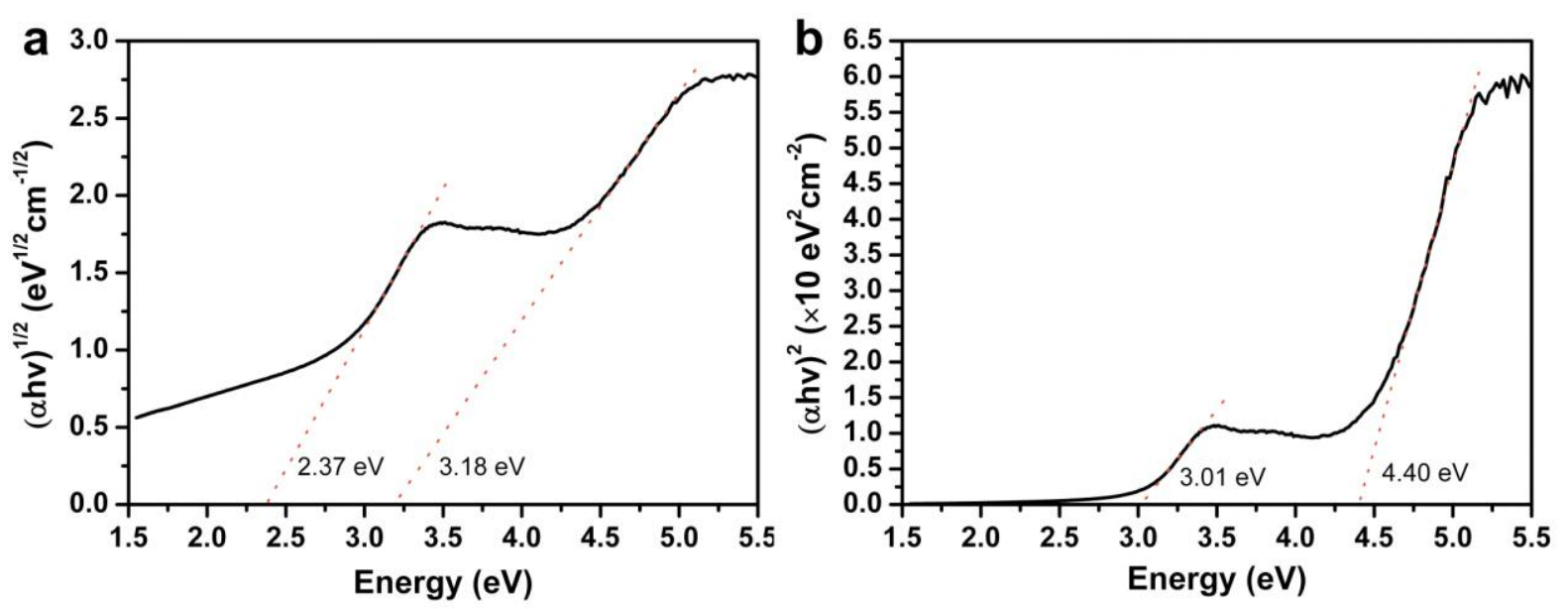

Figure S4. a-b) Plots of $(\alpha h v)^{1 / 2}$ and $(\alpha h v)^{2}$, respectively. The photon energy are calculated from the absorbance measurement. 

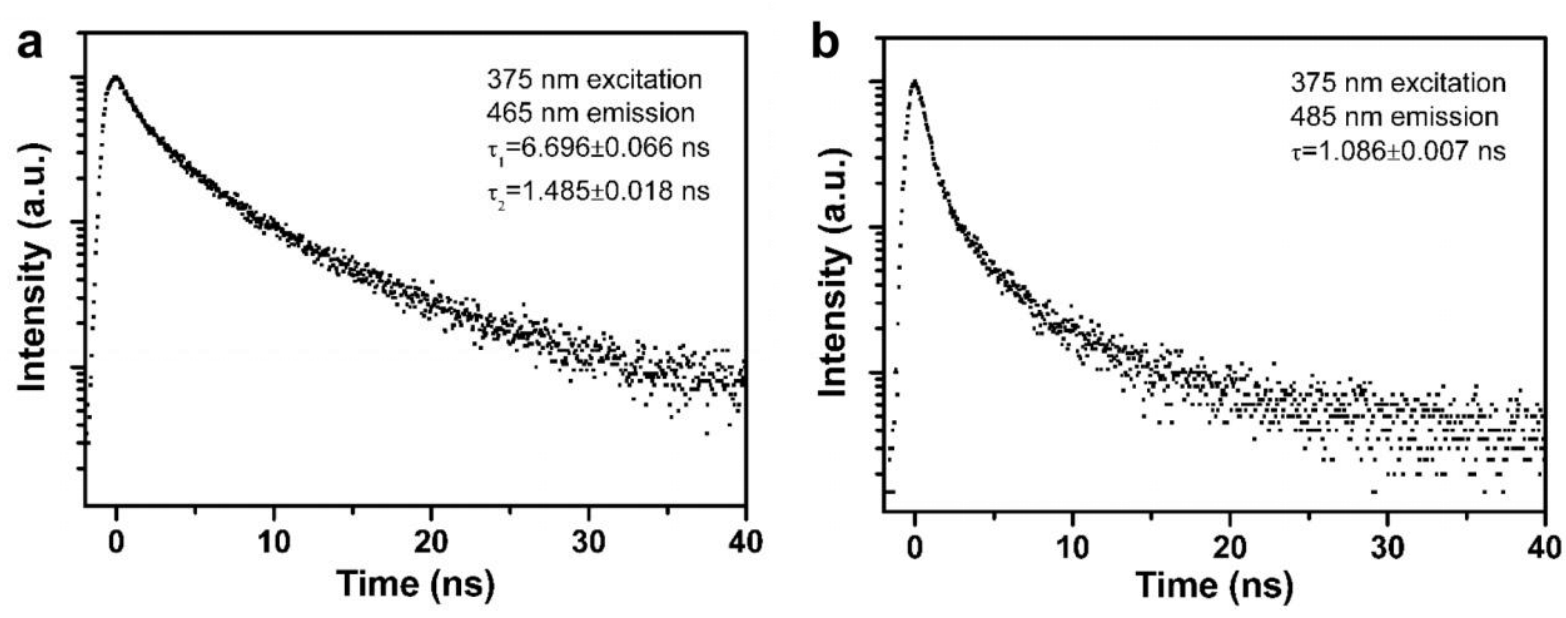

Figure S5. Time-resolved PL spectrum of a) EAC-siloxene, and b) HCl-siloxene (375 nm excitation). The decays are recorded at emission wavelengths of $465 \mathrm{~nm}$ and $485 \mathrm{~nm}$, respectively.

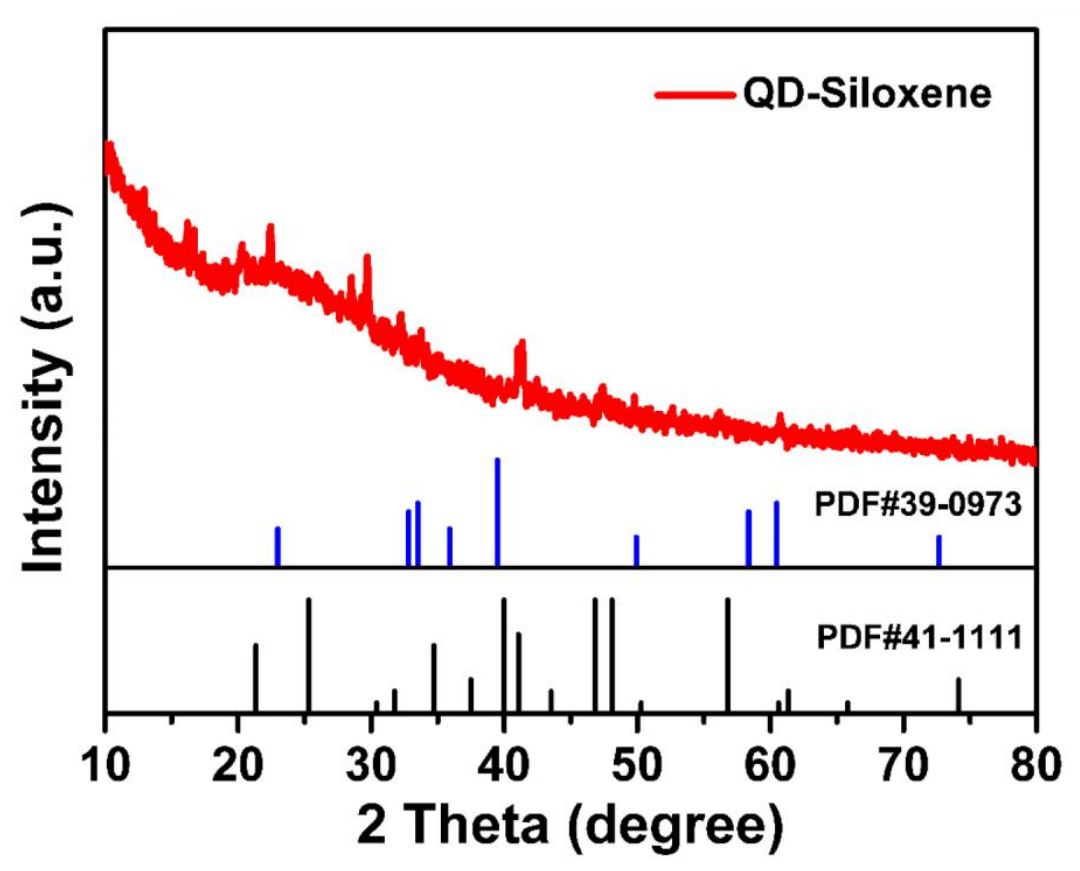

Figure S6. X-ray diffraction pattern of the QD-siloxene nanosheets.

The PDF\#41-1111 and PDF\#39-0973 data is provided in Figure S6 for comparison with the peaks of QD-siloxene. Since the silicene quantum dots are embedded in amorphous oxides and there are defects in the nanosheets, the lattice distortion shifts the diffraction peak positions. 


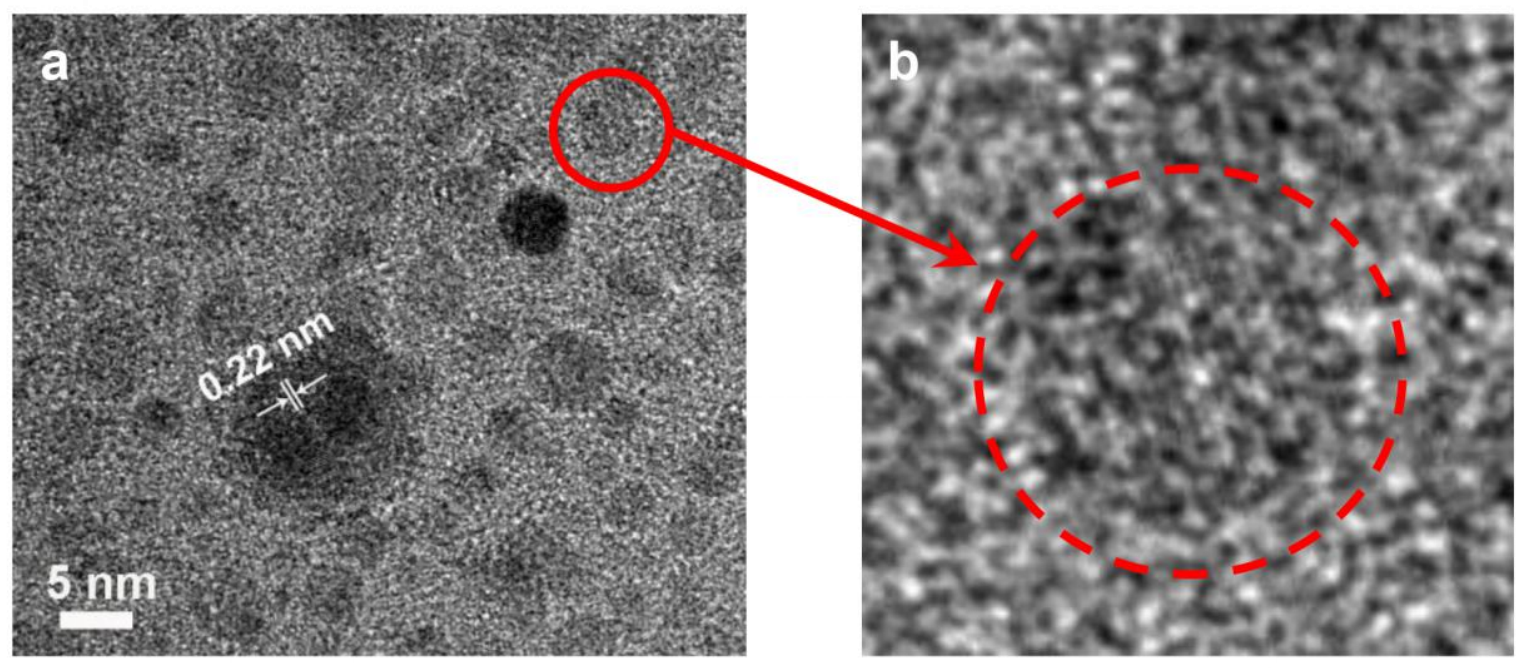

Figure S7. HRTEM images of thin-layered silicene particales with clear edge.

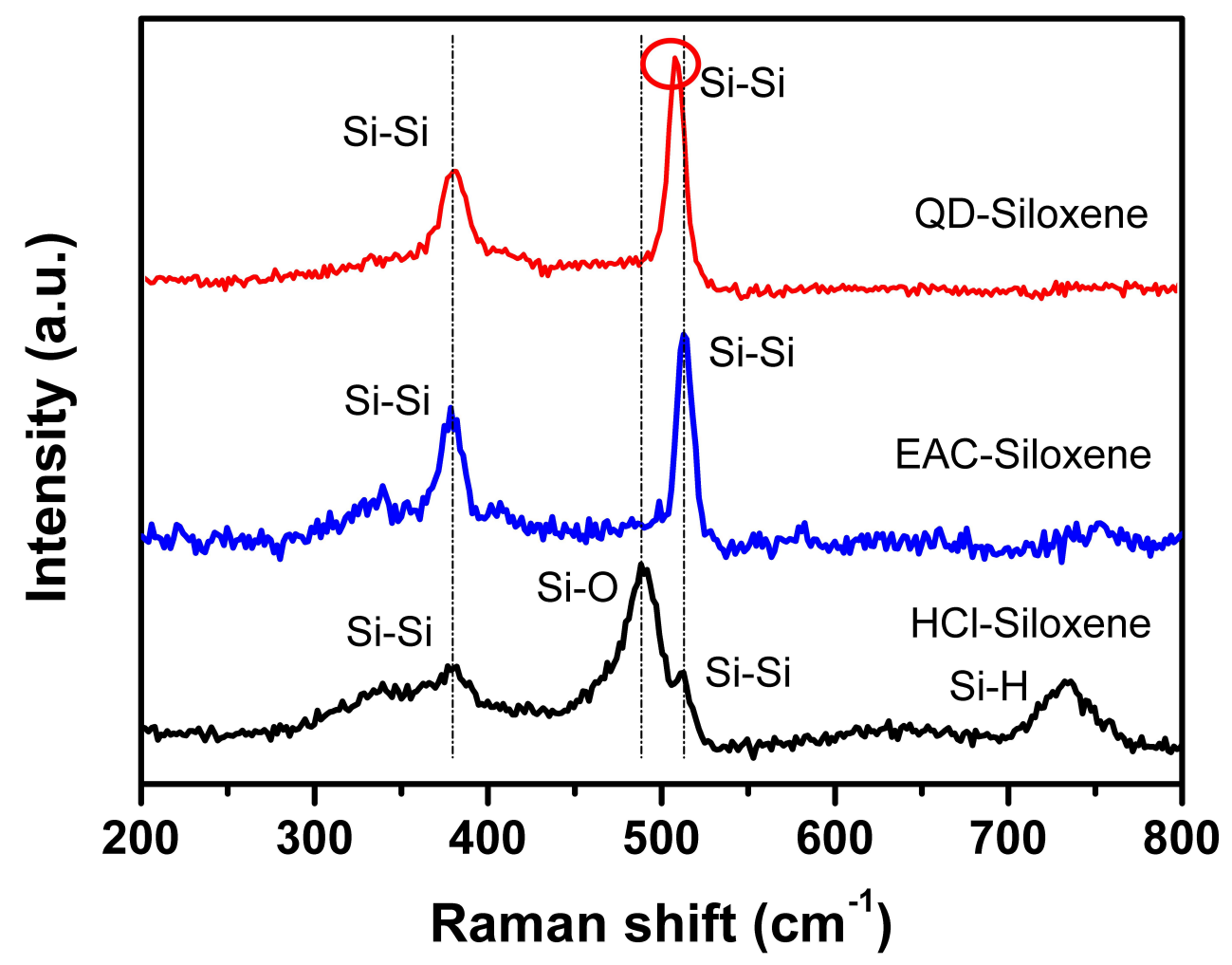

Figure S8. Laser Raman spectra of the products.

The peak at $508 \mathrm{~cm}^{-1}$ has been marked by a red circle. In the Raman spectra, the peak of QDsiloxene (solid, red) is at $508 \mathrm{~cm}^{-1}$, while the peak position of EAC-siloxene (solid, blue) and $\mathrm{HCl}$-siloxene (solid, black) are both at $513 \mathrm{~cm}^{-1}$. 